\title{
Effects of supersymmetric threshold corrections on the Yukawa matrix unification
}

\author{
Mateusz Iskrzyński ${ }^{\mathrm{a}}$ \\ Institute of Theoretical Physics, University of Warsaw, Pasteura 5, 02-093 Warsaw, Poland
}

Received: 19 August 2014 / Accepted: 30 December 2014 / Published online: 4 February 2015

(c) The Author(s) 2015. This article is published with open access at Springerlink.com

\begin{abstract}
We present an updated analysis of the Yukawa matrix unification within the renormalizable R-parityconserving Minimal Supersymmetric Standard Model. It is assumed that the soft terms are non-universal but flavourdiagonal in the super-CKM basis at the GUT scale. Trilinear Higgs-squark-squark $A$-terms can generate large threshold corrections to the Yukawa matrix $\mathbf{Y}^{d}$ at the superpartner decoupling scale. In effect, the $S U(5)$ boundary condition $\mathbf{Y}^{d}=\mathbf{Y}^{e T}$ at the GUT scale can be satisfied. However, such large trilinear terms make the usual Higgs vacuum metastable (though long-lived). We broaden previous studies by including results from the first LHC phase, notably the measurement of the Higgs particle mass, as well as a quantitative investigation of flavour observables.
\end{abstract}

\section{Introduction}

Supersymmetric Grand Unified Theories (SUSY GUTs) have been a topic of multiple studies since the original formulation of the $S U(5)$ model [1]. A successful unification of gauge couplings in the Minimal Supersymmetric Standard Model (MSSM) is a phenomenological triumph of this programme. GUT symmetries are decisively helpful as providers of boundary conditions at the high energy scale, which reduces the dimensionality of the MSSM huge parameter space.

Despite a notable historical success of an approximate bottom-tau Yukawa unification, the absence of such a unification for the first two generations remains a long-standing issue. Modifications of the GUT field content that aimed at solving this problem have been applied already in the non-supersymmetric case [2]. The most exhaustively studied alterations to the boundary conditions at the GUT scale arise from assuming non-negligible effects from higherdimensional operators in the $S U(5)$ model Lagrangian.

a e-mail: mateusz.iskrzynski@fuw.edu.pl
Different mass ratios obtained by such manipulations are reviewed, e.g., in Refs. [3,4].

An important problem of the minimal $S U(5)$ model [1] is the proton decay triggered by the higgsino triplet exchange. Although it remains a non-trivial constraint, several analyses have shown that the theory has not yet been excluded. The proton lifetime can be enhanced by several orders of magnitude by contributions from higher-dimensional operators [5]. Moreover, it has been shown that the tension with experimental results becomes weaker when one uses threeloop renormalisation group equations (RGEs) and two-loop decoupling conditions [6]. In the present paper, we do not restrict ourselves to the minimal model. In particular, we allow the higgsino triplets to acquire superheavy masses from their couplings to additional $\mathbf{5}$ and $\overline{\mathbf{5}}$ fields that do not couple to ordinary matter. No proton decay problem occurs within such a setup, while the Yukawa unification constraint remains the same as in the minimal case.

It has been observed a long time ago that threshold corrections at the superpartner decoupling scale $\mu_{\mathrm{sp}}$ can significantly change or even generate the light fermion masses [7]. This mechanism was applied in the context of grand unification in Ref. [8]. However, in most of the contemporary phenomenological analyses, Yukawa unification has been exhaustively studied only in the third generation case.

A quantitative study that achieved $Y_{S}\left(M_{\mathrm{GUT}}\right)=Y_{\mu}$ $\left(M_{\mathrm{GUT}}\right)$ within renormalizable R-parity-conserving MSSM was performed in Ref. [9]. It included only the threshold corrections coming from gluino and higgsino loops and concluded that a tension arises between the Yukawa unification and flavour observables. That was likely to happen because flavour off-diagonal soft terms were used to generate the Cabibbo angle as well. This analysis was later broadened and simplified to the flavour-diagonal case in Ref. [10]. It provided examples of points in the MSSM parameter space where the $S U(5)$ Yukawa unification was achieved for $\tan \beta \leq 20$. In another article [11] where the leading MSSM 
threshold corrections were investigated, the problem of proton decay was addressed by raising the Higgs soft masses above $30 \mathrm{TeV}$. Examples of including such corrections in non$S U$ (5) models can be found, e.g., in Refs. [12,13].

Our work updates the one of Ref. [10] with a broader range of $\tan \beta$ (reaching 40), inclusion of the contemporary experimental data, as well as a quantitative study of flavour observables. Results from the first phase of the LHC have constrained the superpartner masses and produced the lightest Higgs mass measurement, thus calling for an up-to-date analysis of Yukawa unification.

We shall make use of a corrected account for chirally enhanced threshold corrections to fermion masses in the MSSM. It was summarised in Ref. [14] and earlier published as parts of other analyses [15-17]. However, the two-loop effects computed and described in Ref. [18] are not included in the present work. It might be interesting to study their effect in the future even though they are unlikely to affect our final conclusions.

The article is organised as follows. In Sect. 2, our phenomenological scenario within the MSSM is described. Sect. 3 is devoted to analysing threshold corrections to the Yukawa couplings, and to studying in what manner their unification depends on the most important variables. In Sect. 4, particular examples of points in the MSSM parameter space with Yukawa unification are given. The impact of large $A$ terms on flavour observables is examined in Sect. 5, whereas a correlation with the vacuum metastability is explained in Sect. 6.

\section{The model}

\subsection{Relevant aspects of the SUSY SU(5) GUT}

The SM gauge group is a subgroup of $S U(5)$. A standard embedding of the MSSM superfields $Q, U, D, L, E$ into the five- and ten-dimensional representations of $S U(5)$ is given by

$$
\begin{array}{r}
\underbrace{\left(\overline{\mathbf{3}}, \mathbf{1}, \frac{1}{3}\right)}_{D} \oplus \underbrace{\left(\mathbf{1}, \mathbf{2},-\frac{1}{2}\right)}_{L}=\underbrace{\overline{\mathbf{5}}}_{\Psi_{\overline{5}}}, \\
\underbrace{\left(\mathbf{3}, \mathbf{2}, \frac{1}{6}\right)}_{Q} \oplus \underbrace{\left(\overline{\mathbf{3}}, \mathbf{1},-\frac{2}{3}\right)}_{U} \oplus \underbrace{(\mathbf{1}, \mathbf{1}, 1)}_{E}=\underbrace{\mathbf{1 0}}_{\Psi_{10}},
\end{array}
$$

where the hypercharges have been displayed in the conventional SM normalisation. We are going to consider $S U(5)$ GUTs whose Yukawa terms in the superpotential read [1]

$\mathcal{W} \ni \Psi_{10} \mathbf{Y}^{d e} \Psi_{\overline{5}} H_{\overline{5}}+\Psi_{10} \mathbf{Y}^{u} \Psi_{10} H_{5}$, where $H_{5}$ and $H_{5}$ are the two Higgs superfields that couple to matter. Masses of the known fermions are thus determined by only two independent $3 \times 3$ matrices $\mathbf{Y}^{d e}$ and $\mathbf{Y}^{u}$. Below the gauge unification scale $M_{\mathrm{GUT}} \simeq 2 \times 10^{16} \mathrm{GeV}$, the model reduces to the MSSM with the superpotential given by

$\mathcal{W}_{M S S M}=Q \mathbf{Y}^{u} U H_{u}+Q \mathbf{Y}^{d} D H_{d}+L \mathbf{Y}^{e} E H_{d}+\mu H_{d} H_{u}$.

Thence, $\mathbf{Y}^{d}$ and $\mathbf{Y}^{e T}$ are equal at the matching scale $M_{\mathrm{GUT}}$, up to a basis redefinition, and up to threshold corrections at this scale.

Unification constraints for $\mathbf{Y}^{d}$ and $\mathbf{Y}^{e}$ take the simplest form in a basis where the superpotential flavour mixing has been entirely included in $\mathbf{Y}^{u}$, while $\mathbf{Y}^{d}$ and $\mathbf{Y}^{e}$ are real and diagonal. Then we just require equality of the diagonal entries:

$\mathbf{Y}_{i i}^{d} \stackrel{!}{=} \mathbf{Y}_{i i}^{e}, \quad i=1,2,3$.

Below $M_{\mathrm{GUT}}$, the relation between $\mathbf{Y}^{d}$ and $\mathbf{Y}^{e}$ is affected by the RGE and, most importantly, by the threshold corrections at $\mu_{\mathrm{sp}}$ that strongly depend on the soft supersymmetry breaking terms. These terms include the gaugino masses $M_{\tilde{B}}, M_{\tilde{W}}$, $M_{\tilde{g}}$, soft scalar masses $m_{\tilde{q}}^{2}, m_{\tilde{u}}^{2}, m_{\tilde{d}}^{2}, m_{\tilde{l}}^{2}, m_{\tilde{e}}^{2}, m_{h_{d}}^{2}, m_{h_{u}}^{2}$, as well as the bi- and trilinear interactions of the higgses and sfermions (squarks and sleptons)

$\mathcal{L}_{\text {soft }} \ni \tilde{q} \mathbf{A}^{u} \tilde{u} h_{u}+\tilde{q} \mathbf{A}^{d} \tilde{d} h_{d}+\tilde{l} \mathbf{A}^{e} \tilde{e} h_{d}+B \mu h_{d} h_{u}+$ h.c.

Our assumptions concerning the soft supersymmetry breaking terms are outlined in the next section.

\subsection{Choice of the parameters}

In order to approach the question of unifying Yukawa couplings by an appropriate choice of the MSSM parameters, we assume the validity of its RGEs up to $M_{\mathrm{GUT}}$ where the $S U(5)$ boundary conditions are imposed.

The phenomenological motivation behind the discussed scenario within renormalizable R-parity-conserving MSSM is to achieve Yukawa unification and fulfil experimental conditions in the simplest manner, constraining as few parameters as possible. To independently influence the ratios $\mathbf{Y}_{i i}^{d} / \mathbf{Y}_{i i}^{e}$ for all the three families, one needs to adjust at least three real parameters.

Diagonal entries of the trilinear $\mathbf{A}^{d e}$-terms in the superCKM basis (which we use throughout the article) can well serve this purpose, as they have a strong influence on the relevant threshold corrections. Moreover, to obtain a correct mass of the lightest Higgs boson for given sparticle masses, one has to adjust $\mathbf{A}_{33}^{u}$ that governs the stop mixing [19]. 
Table 1 Standard Model parameters [27] used in our numerical calculations. The light $(u, d, s)$ quark masses are $\overline{\mathrm{MS}}$-renormalised at $2 \mathrm{GeV}$

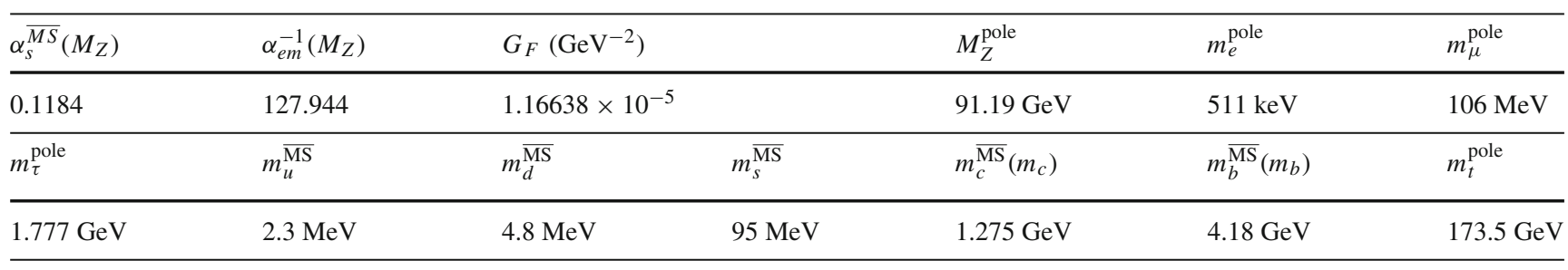

Both the Higgs soft mass terms and $\tan \beta=\frac{v_{u}}{v_{d}}$, which we employ to parametrise the Higgs sector, are unconstrained by the $S U(5)$ unification conditions and can serve other phenomenological purposes. As far as the gaugino and the soft sfermion masses at the GUT scale are concerned, we restrict ourselves here to the simplest choice of a common gaugino mass $M_{1 / 2}$ and a universal soft mass $m_{0}$ for all the sfermions (but not the Higgs doublets). Such a choice reduces the number of free parameters and makes the analysis transparent. However, it is by no means necessary for achieving the Yukawa matrix unification.

In total, our scenario has nine free parameters: $\tan \beta, M_{1 / 2}$, $m_{0}, m_{h_{u}}, m_{h_{d}}, \mathbf{A}_{11}^{d e}, \mathbf{A}_{22}^{d e}, \mathbf{A}_{33}^{d e}, \mathbf{A}_{33}^{u}$.

\subsection{Tools}

A standard numerical procedure that for a given parameter set leads to a full spectrum of the MSSM can be summarised as follows. The renormalisation group equations of MSSM are solved by an iterative algorithm that interpolates between various scales at which the parameter values are assumed. The boundary with the SM (i.e. the scale $\mu_{\mathrm{sp}}$ ) is currently set by most of the public programs to be at $M_{Z}$. Such a choice has considerable disadvantages, one of which is excluding too many parameter points from the analysis. For instance, some fields become formally tachyonic only well below their actual mass scale but above $M_{Z}$, which is still acceptable, though most programs usually reject such points.

Minimisation of the MSSM scalar potential is performed at the scale $M_{\text {SUSY }}=\sqrt{m_{\tilde{t}_{1}} m_{\tilde{t}_{2}}}$, where the scale dependence of the electroweak breaking conditions is relatively mild.

A recent article [20] has shown that the contemporary spectrum generators find only one of the potentially many models corresponding to a given set of parameters that are specified at multiple energy scales. In particular, it affects the cMSSM dark matter analyses [21]. However, this fact hardly matters for our present investigation because we only search for sample regions in the parameter space where the Yukawa unification constraint is satisfied.

For the purpose of the current analysis, we have modified SOFTSUSY 3.3.8 [22] that distinguishes itself among other spectrum generators by possessing a technical documentation. We implemented threshold corrections to the first and second family Yukawa couplings as well as to the CKM matrix, given in Ref. [14], which SOFTSUSY was lacking at the moment of writing.

Our input values of the SM parameters are collected in Table 1. Flavour observables are calculated with the help of SUSY_FLAVOR V2.10 [23]. This code evaluates the renormalised MSSM Yukawa matrices and obtains the proper CKM matrix also according to the prescriptions of Ref. [14], taking the previously determined soft parameters as input.

In our scan, which produced regions consistent with the Yukawa unification, we use the BayesFITSv3.2 [24,25] numerical package that interfaces several publicly available codes. Except the above-mentioned programs, it uses MultiNest v2 . 7 [26] which enables a fast and efficient Markov Chain Monte Carlo (MCMC) scanning according to a pre-defined likelihood function. For the $S U(5)$ boundary condition in Eq. (5), we assume a Gaussian likelihood distribution

$$
\mathcal{L}_{\mathrm{Yuk}}=\sum_{i=1,2,3} \exp \left[-\left(1-Y_{i i}^{e}\left(M_{\mathrm{GUT}}\right) / Y_{i i}^{d}\left(M_{\mathrm{GUT}}\right)\right)^{2} / 2 \sigma_{\mathrm{Yuk}}^{2}\right]
$$

with $\sigma_{\text {Yuk }}$ set to $10 \%$ to allow for deviations from the exact unification condition.

\section{Analysis of threshold corrections to the Yukawa matrices at $\mu_{\mathrm{sp}}$}

Given our choice of the GUT-scale parameters, the only source of flavour violation at this scale is the Yukawa matrix $\mathbf{Y}^{u}$. Since it affects the RGE for the remaining parameters, neither $\mathbf{Y}^{d}$ nor the soft terms are going to remain strictly flavour-diagonal below $M_{\mathrm{GUT}}$. However, the corresponding flavour violation is going to be given by the CKM matrix and remain genuinely small. While such flavour violation is taken into account in our numerical study, we shall neglect it for simplicity in the following discussion where large corrections to the flavour-diagonal terms are of main interest. Within such an approximation, it is sufficient to consider only real diagonal Yukawa matrices $\mathbf{Y}^{d} \equiv \operatorname{diag}\left(Y_{d}, Y_{s}, Y_{b}\right)$ and $\mathbf{Y}^{e} \equiv \operatorname{diag}\left(Y_{e}, Y_{\mu}, Y_{\tau}\right)$ at all the renormalisation scales. 


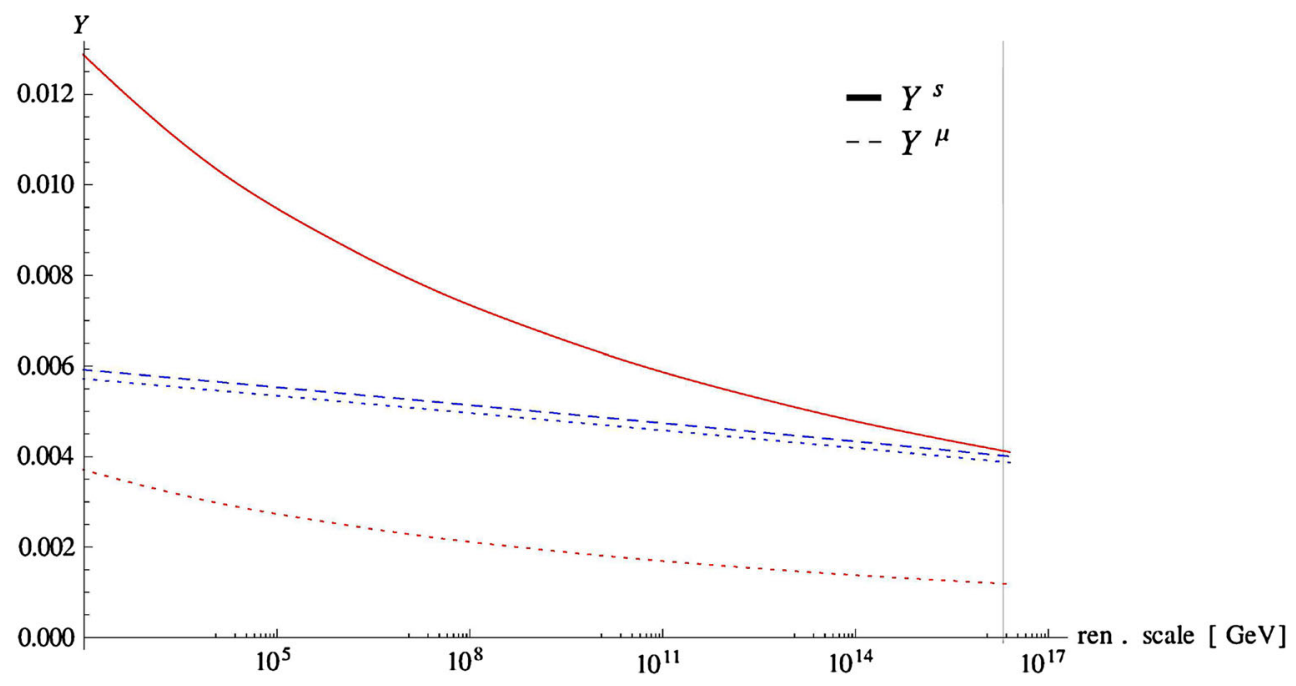

Fig. 1 RGE running of $Y_{s}$ (red) and $Y_{\mu}$ (blue) between $\mu_{\mathrm{sp}}$ and $M_{\mathrm{GUT}}$ for a sample point in the MSSM parameter space. Dotted lines describe a situation with vanishing threshold corrections at $\mu_{\mathrm{sp}}$. For the solid and dashed lines, the threshold corrections have been adjusted to achieve unification at the GUT scale

gram in Fig. 2. It can be used to adjust the threshold correction and to achieve the Yukawa unification for given values of other parameters.

In the following, we shall illustrate how the threshold corrections to the Yukawa couplings at $\mu_{\mathrm{sp}}$

$\delta Y_{x} \equiv \frac{v_{d} Y_{x}^{\mathrm{MSSM}}-m_{x}^{\mathrm{SM}}}{m_{x}^{\mathrm{SM}}}, \quad x=d, s, b, e, \mu, \tau$,

as well as the ratio $\mathbf{Y}_{i i}^{d} / \mathbf{Y}_{i i}^{e}$ at the GUT scale depend on the most important parameters of the model. Using the point no. 3 in Table 2 (Sect. 4) as a reference, we have varied only two parameters at a time, which gives an estimate of the shape of the relation in the vicinity of the considered point. We concentrate on the cases of the second and third family, as the first and second ones are qualitatively similar.

In Figs. 3, 4 and 5, we show only the points fulfilling all the basic phenomenological requirements, in particular that the Higgs vacuum is a local minimum of the scalar potential, 1 and that no Landau poles arise below $M_{\mathrm{GUT}}$. White regions in the plots mean that either one of above conditions was not fulfilled, or that SOFTSUSY rejected the point as its iterative algorithm had not converged.

Starting from the largest couplings, we notice that three parameters play a crucial role in the case of bottom-tau unification: $\mathbf{A}_{33}^{d e}, \mu$ and $m_{0}$ (which for given $M_{1 / 2}$ governs masses of the third family sfermions). Non-universal sfermion masses, independent for each family, could grant additional freedom to our model. Although they are not necessary to achieve Yukawa unification, relaxation of the uni-

$\overline{1}$ No scalar tachyons appear in the spectrum. 

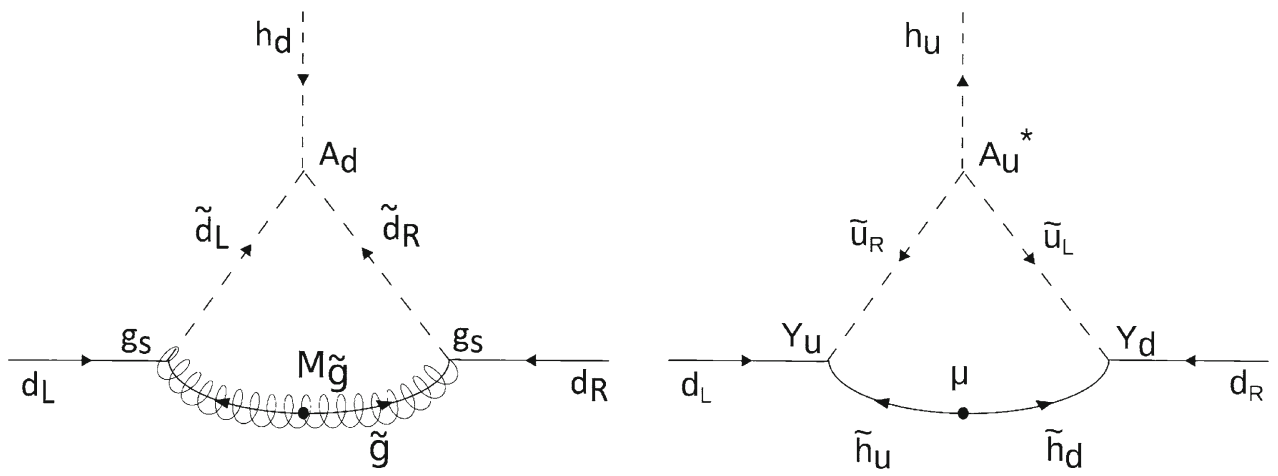

Fig. 2 Examples of diagrams that describe threshold corrections to the Yukawa couplings at $\mu_{\mathrm{sp}}$

Table 2 Examples of points with a successful Yukawa unification. They are given by their defining sets of MSSM parameters: tan $\beta$, common gaugino mass $M_{1 / 2}$, common sfermion mass $m_{0}$, soft masses of Higgs doublets $m_{h_{u}}$ and $m_{h_{d}}$, soft trilinear couplings $\mathbf{A}_{i i}$ ( $M_{\mathrm{GUT}}$ )

\begin{tabular}{|c|c|c|c|c|c|c|c|c|c|}
\hline & $\tan \beta$ & $\frac{M_{1 / 2}}{\mathrm{GeV}}$ & $\frac{m_{0}}{M_{1 / 2}}$ & $\frac{m_{h_{u}}}{m_{0}}$ & $\frac{m_{h_{d}}}{m_{0}}$ & $\frac{\mathbf{A}_{11}^{d e}}{M_{1 / 2}}$ & $\frac{\mathbf{A}_{22}^{d e}}{M_{1 / 2}}$ & $\frac{\mathbf{A}_{33}^{d e}}{M_{1 / 2}}$ & $\frac{\mathbf{A}_{33}^{u}}{M_{1 / 2}^{u}}$ \\
\hline 1 & 7.47 & $1,032.27$ & 2.28 & 0.49 & 2.35 & -0.008 & 0.63 & 1.63 & -2.13 \\
\hline 2 & 16.4 & 815.081 & 1.72 & 0.37 & 2.46 & -0.017 & 0.89 & 1.30 & -1.23 \\
\hline 3 & 30.3 & $2,733.63$ & 1.67 & 0.19 & 1.79 & -0.037 & 1.22 & 1.04 & 1.96 \\
\hline 4 & 40.4 & $2,663.45$ & 1.72 & 0.26 & 2.58 & -0.055 & 1.53 & 1.65 & 1.56 \\
\hline
\end{tabular}

versality could facilitate finding points with even higher $\tan \beta$ than presented in the next section.

Values of the ratio $Y_{b} / Y_{\tau}$ at $M_{\mathrm{GUT}}$ are presented in Fig. 3 as functions of $\mathbf{A}_{33}^{d e}$ and $m_{0}$. The equality of $Y_{b}$ and $Y_{\tau}$ at this scale in general might demand an adjustment of all the parameters because excluded points tightly surround the allowed region.

In the second family case, unification of $Y_{S}$ and $Y_{\mu}$ is usually possible by a manipulation of just one parameter, namely $\mathbf{A}_{22}^{\text {de }}$, despite the fact that it influences both Yukawa couplings. For the second family, $\mu$ has little influence on the unification in the considered region because the higgsino loop gives a much smaller contribution, due to $m_{c} \ll m_{t}$.

The ratio $Y_{s} / Y_{\mu}$ at $M_{\mathrm{GUT}}$ plotted in Fig. 4 against $m_{0}$ and $\mathbf{A}_{22}^{d e}$ shows that a large value of $\mathbf{A}_{22}^{d e}$ is required to achieve unification. The corresponding values of $\mathbf{A}_{22}^{d} / \tilde{m}_{2}$ at $M_{\text {SUSY }}$ are shown in Fig. 4. Such ratios will be relevant for our discussion of the vacuum metastability in Sect. 6 . Here, $\tilde{m}_{i}$ are defined by

$\tilde{m}_{i}=\sqrt{\frac{m_{\tilde{q}_{i}}^{2}+m_{\tilde{d}_{i}}^{2}+m_{H_{d}}^{2}}{3}}$.

Unification of the down-quark and electron Yukawa couplings is illustrated in Fig. 5. It is the simplest case, because the necessary adjustment of the respective $A$-term neither triggers any phenomenological problems nor influences any parameters that are relevant for other families.

\section{Regions with successful SU(5) Yukawa matrix unification}

In Figs. 6 and 7 as well as in Table 2, we present sample parameter-space regions and benchmark points where a proper Yukawa matrix unification has been achieved in our setup. In selecting these regions and points, we aimed at fulfilling the unification constraints and reproducing the lightest Higgs particle mass (up to the theoretical uncertainty of $3 \mathrm{GeV}$ ) for a broad range of $\tan \beta$. We have chosen the sparticle masses so that the gluino is heavy enough to have evaded the current bounds, but could possibly be detected in the second LHC phase.

The plots in Figs. 6 and 7 show points investigated in our MCMC scans performed for three $\tan \beta$ intervals: $[5,20],[15,30]$ and $[30,45]$. Different colours are used to indicate successful Yukawa matrix unification either for all the three families or for some of them only. We observe that Yukawa unification for all the three generations can be achieved for a wide range of $\tan \beta$. Generically, larger values of the $A$-terms are necessary for larger $\tan \beta$ because the down-quark Yukawa couplings (and thus the required threshold corrections) scale proportionally to $\tan \beta$. For this reason, finding acceptable points for larger values of $\tan \beta$ in each random scan required collecting much more statistics.

Tables 2, 3, 4 and 5 contain information on the input parameters and particle spectra in four sample points with a proper Yukawa matrix unification. Table 3 shows the cor- 

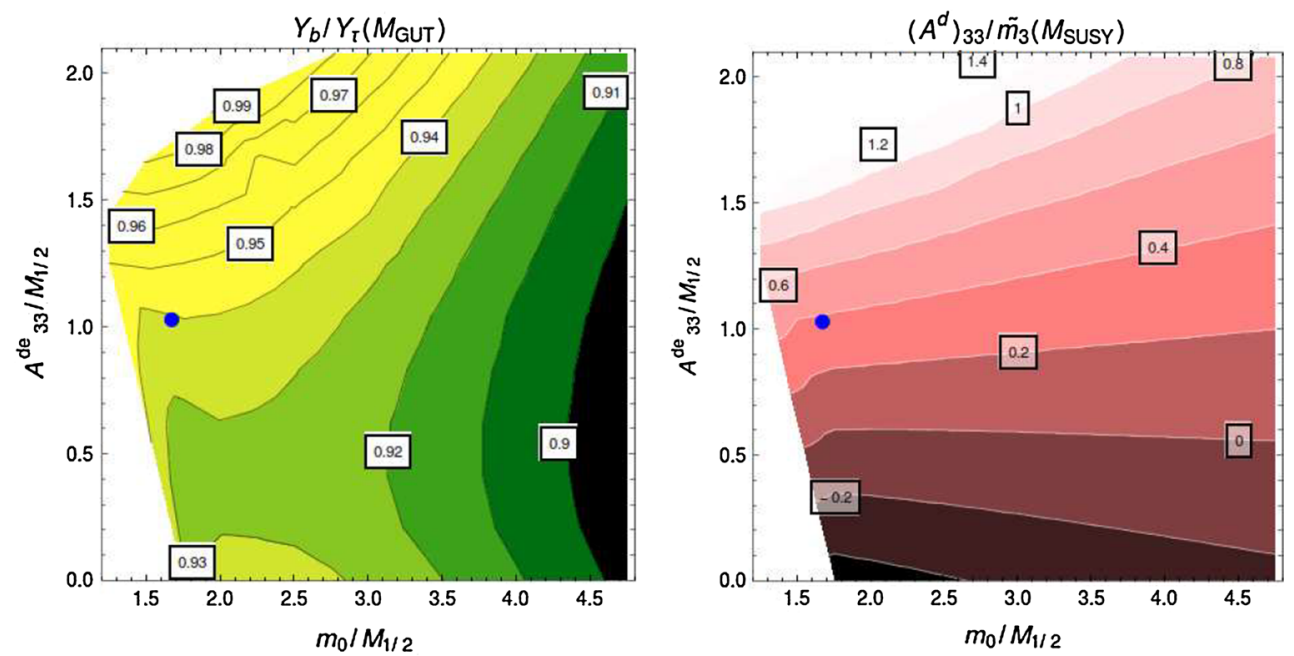

Fig. 3 Left The ratio $Y_{b} / Y_{\tau}$ presented as a function of $\mathbf{A}_{33}^{d e}$ and $m_{0}$. Right The corresponding values of $\mathbf{A}_{33}^{d} / \tilde{m}_{3}$ at $M_{\text {SUSY }}$. They are shown around point 3 from Table 2 (marked by a blue dot). Both $\mathbf{A}_{33}^{d e}$ and $m_{0}$ are normalised to $M_{1 / 2}$ which equals around $815 \mathrm{GeV}$ at that point
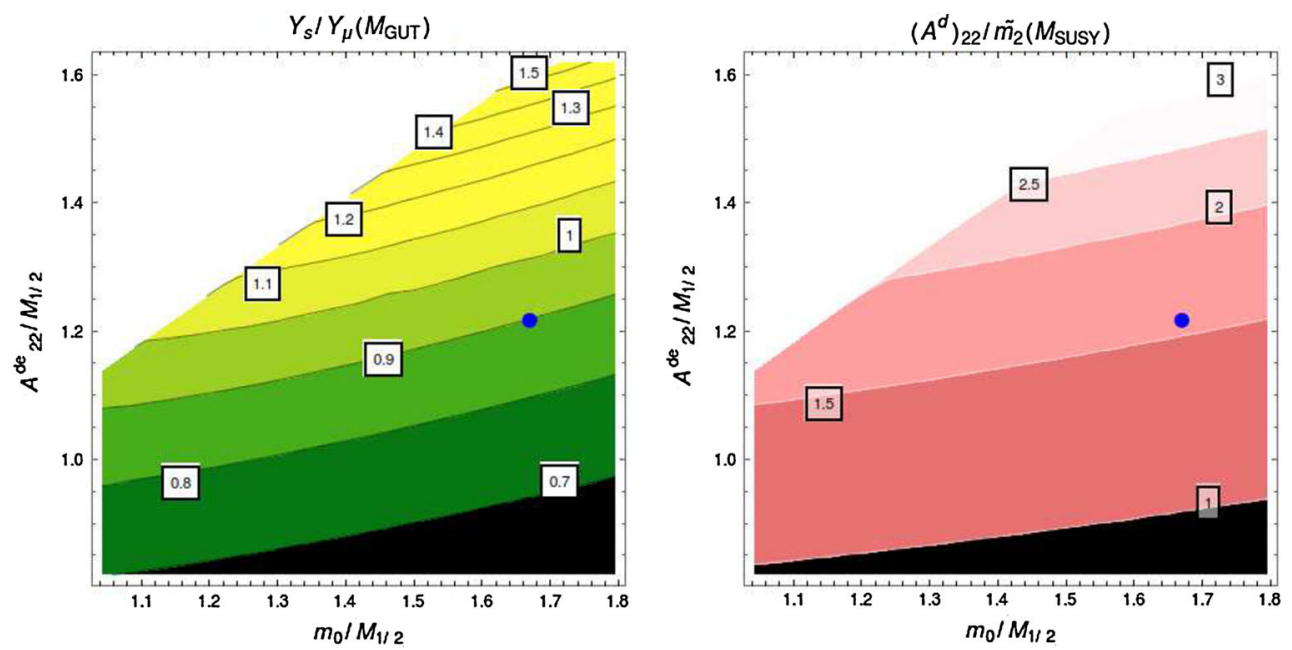

Fig. 4 Left The ratio $Y_{s} / Y_{\mu}$ presented as a function of $\mathbf{A}_{22}^{d e}$ and $m_{0}$. Right The corresponding values of $\mathbf{A}_{22}^{d} / \tilde{m}_{2}$ at $M_{\mathrm{SUSY}}$. They are shown around point 3 from Table 2 (marked by a blue dot)

responding SUSY-scale threshold corrections, as defined in Eq. (10). In addition, we give the GUT-scale ratios $\frac{Y_{i}^{d}}{Y_{i}^{e}}$ which parametrise the unification quality. Their (small) deviations from unity determine sizes of the necessary GUTscale threshold corrections. Finally, we also present the ratios $\frac{m_{h_{d}}}{m_{0}}$ and $\frac{m_{h_{u}}}{m_{0}}$ at the GUT scale that quantify departures from the scalar mass universality for each of the points.

\section{Flavour observables}

In this section, we discuss the impact of large $A$-terms on flavour observables. The MSSM scenario we consider does not include any sources of flavour- and $\mathrm{CP}$ - violation at $M_{\mathrm{GUT}}$ other than the CKM matrix. Therefore, flavour off-diagonal entries of the soft terms remain small, as they arise solely from the RGE running. ${ }^{2}$

In the following, we shall illustrate how the flavour observables change when the $A$-terms grow from 0 to $150 \%$ of the value that is necessary for the Yukawa unification $\mathbf{Y}^{d}\left(M_{\mathrm{GUT}}\right)=\mathbf{Y}^{e T}\left(M_{\mathrm{GUT}}\right)$ to take place. Among the observables calculable with the help of SUSY_FLAVOR V2.10, only three turn out to be significantly altered:

$$
\begin{gathered}
\mathcal{B}_{\gamma} \equiv \mathcal{B}\left(\bar{B} \rightarrow X_{s} \gamma\right), \quad \overline{\mathcal{B}}_{s \mu} \equiv \overline{\mathcal{B}}\left(B_{s} \rightarrow \mu^{+} \mu^{-}\right) \\
\text {and } \overline{\mathcal{B}}_{d \mu} \equiv \overline{\mathcal{B}}\left(B_{d} \rightarrow \mu^{+} \mu^{-}\right)
\end{gathered}
$$

$\overline{2 \text { SOFTSUSY }}$ 3.3.8 assumes that all the MSSM parameters are real, i.e. it neglects the $\mathrm{CP}$-violating phases. A separate numerical evaluation of the soft term imaginary parts has been performed with the help of SPheno 3.3.3 [28,29]. No observable impact on CP-violating observables has been found for the MSSM parameter-space points discussed in the previous section. 

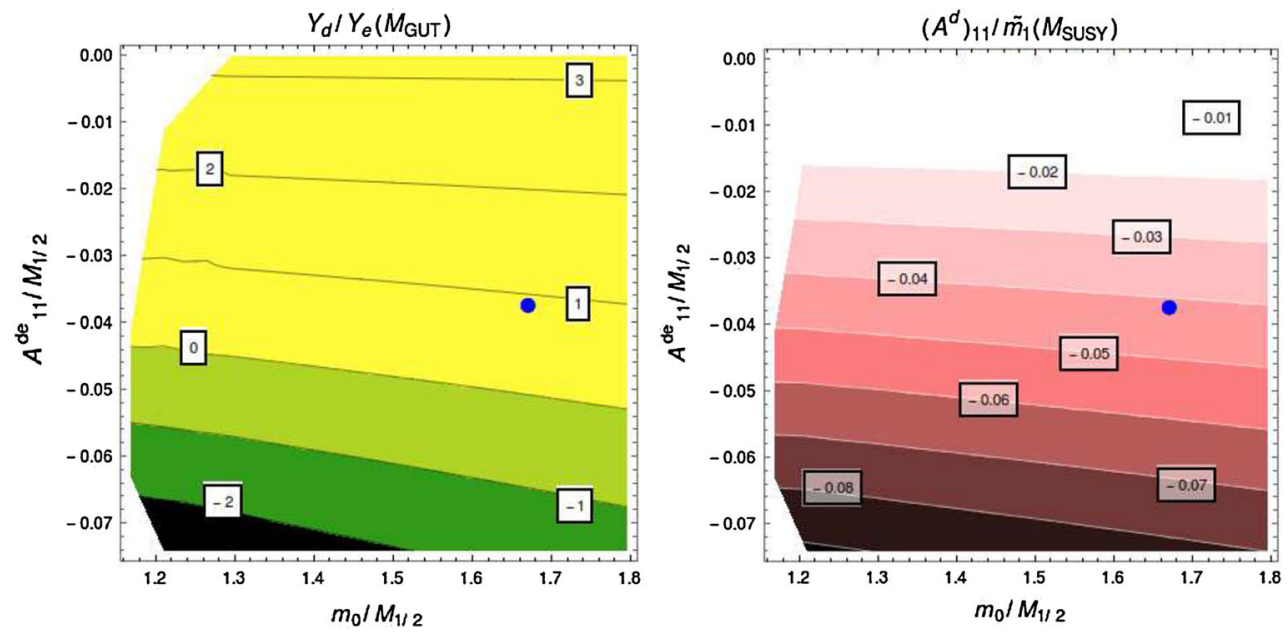

Fig. 5 Left The ratio $Y_{d} / Y_{e}$ as a function of $\mathbf{A}_{11}^{d e}$ and $m_{0}$. Right The corresponding values of $\mathbf{A}_{11}^{d} / \tilde{m}_{1}$ at $M_{\mathrm{SUSY}}$. They are shown around point 3 from Table 2 (marked by a blue dot)

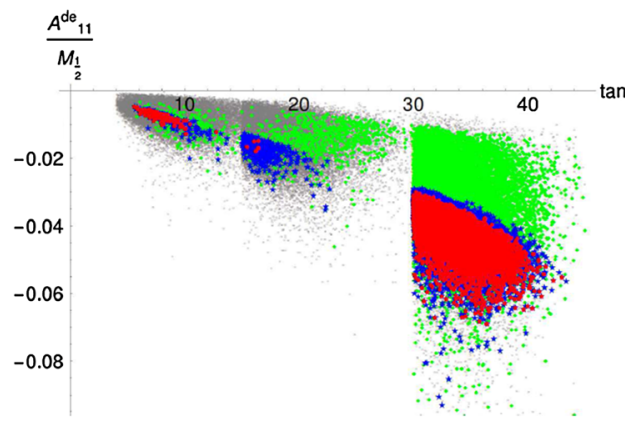

Fig. 6 Left points gathered in three of our MCMC scans ( grey), shown in the $\tan \beta \times\left(\mathbf{A}_{11}^{d e} / M_{1 / 2}\right)$ plane. For some of them, the respective Yukawa couplings get unified within a $10 \%$ bound and the Higgs boson mass prediction lies in the interval $[122.5,128.5] \mathrm{GeV}$ : green diamonds

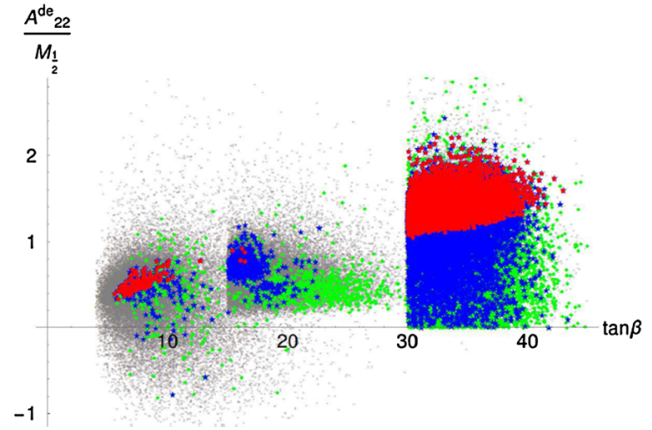

mark the $b-\tau$ unification, blue stars fulfil also the $d-e$ one, while red circles include also the $s-\mu$ one (i.e. the full Yukawa matrices get unified). Right the same data projected onto the $\tan \beta \times\left(\mathbf{A}_{22}^{\text {de }} / M_{1 / 2}\right)$ plane
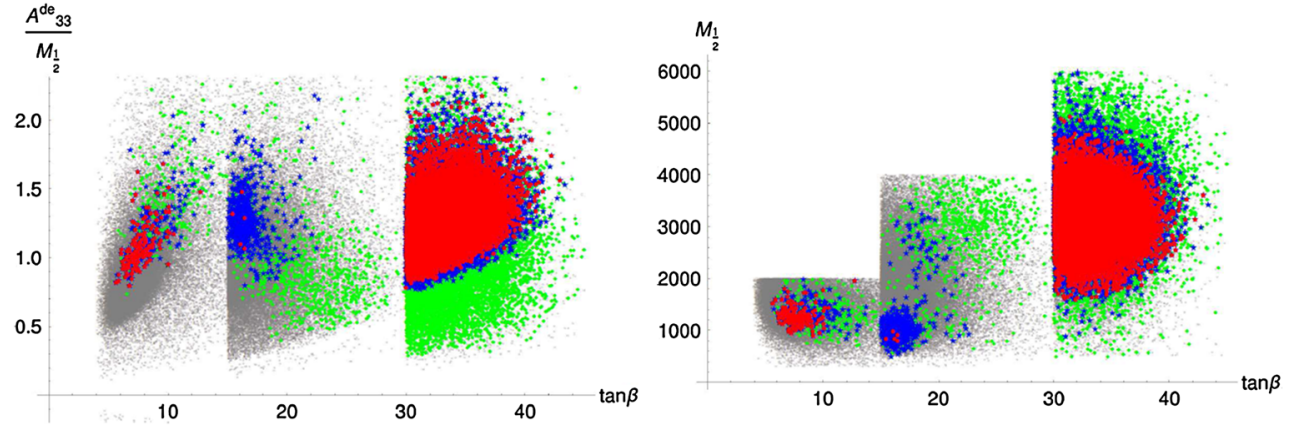

Fig. 7 The same data as in Fig. 6 projected onto the $\tan \beta \times\left(\mathbf{A}_{33}^{d e} / M_{1 / 2}\right)(l e f t)$ and $\tan \beta \times M_{1 / 2}($ right $)$ planes

Table 3 Values of the threshold corrections and other characteristics of the points from Table 2 (see the text)

\begin{tabular}{lllllll}
\hline & $\delta Y_{d}$ & $\delta Y_{s}$ & $\delta Y_{b}$ & $\frac{Y_{d}}{Y_{e}}$ & $\frac{Y_{s}}{Y_{\mu}}$ & $\frac{Y_{b}}{Y_{\tau}}$ \\
\hline 1 & -0.6 & 2.4 & 0.19 & 1.10 & 0.94 & 0.97 \\
2 & -0.66 & 2.3 & 0.089 & 0.95 & 0.91 & 0.90 \\
3 & -0.69 & 2.1 & 0.085 & 0.92 & 0.90 & 0.94 \\
4 & -0.66 & 2.1 & 0.13 & 1.01 & 0.92 & 1.08 \\
\hline
\end{tabular}


Table 4 Masses of selected sfermions (in $\mathrm{GeV}$ ) corresponding to the points from Table 2. In the case of the second generation, where the left-right mixing is negligible, mass eigenstates are labelled according to their largest interaction eigenstate component

\begin{tabular}{lllllllllll}
\hline & $m_{\tilde{s}_{L}}$ & $m_{\tilde{s}_{R}}$ & $m_{\tilde{\mu}_{L}}$ & $m_{\tilde{\mu}_{R}}$ & $m_{\tilde{t}_{1}}$ & $m_{\tilde{t}_{2}}$ & $m_{\tilde{b}_{1}}$ & $m_{\tilde{b}_{2}}$ & $m_{\tilde{\tau}_{1}}$ & $m_{\tilde{\tau}_{2}}$ \\
\hline 1 & 2,031 & 1,856 & 1,433 & 1,433 & 1,328 & 1,649 & 1,603 & 1,652 & 1,130 & 1,185 \\
2 & 3,049 & 2,958 & 2,579 & 2,579 & 1,542 & 2,085 & 2,068 & 2,154 & 1,916 & 2,011 \\
3 & 5,999 & 4,944 & 3,752 & 3,752 & 5,596 & 4,933 & 5,620 & 5,698 & 3,937 & 4,180 \\
4 & 5,700 & 4,290 & 3,714 & 3,714 & 4,902 & 4,951 & 4,904 & 3,984 & 2,658 & 2,658 \\
\hline
\end{tabular}

Table 5 Masses of the gluino, neutralinos, charginos, pseudoscalar $A_{0}$ and the value of $\mu$ parameter (in $\mathrm{GeV}$ ) corresponding to the points from Table 2

\begin{tabular}{rrrrrrrrrr}
\hline & $m_{\tilde{g}}$ & $m_{\chi_{1}^{0}}$ & $m_{\chi_{2}^{0}}$ & $m_{\chi_{3}^{0}}$ & $m_{\chi_{4}^{0}}$ & $m_{\chi_{1}^{ \pm}}$ & $m_{\chi_{2}^{ \pm}}$ & $m_{A_{0}}$ & $\mu$ \\
\hline 1 & 2,350 & 453 & 865 & 2,688 & 2,688 & 865 & 2,689 & 5,192 \\
2 & 1,858 & 350 & 670 & 1,626 & 1,626 & 670 & 1,628 & 2,981 \\
3 & 5,719 & 1,223 & 2,269 & 4,529 & 4,530 & 2,270 & 4,531 & 5,523 & $-1,626$ \\
4 & 5,545 & 1,189 & 2,205 & 3,690 & 3,691 & 2,205 & 3,692 & 6,958 & $-3,686$ \\
\hline
\end{tabular}

Moreover, the only A-term component they noticeably depend on is $\mathbf{A}_{33}^{d e}$. Another important parameter to which these observables are sensitive is $\tan \beta$.

In Fig. 8, we show the dependence of $\delta \mathcal{B}_{\gamma} \equiv\left(\mathcal{B}_{\gamma}^{\mathrm{MSSM}}-\right.$ $\left.\mathcal{B}_{\gamma}^{\mathrm{SM}}\right) / \mathcal{B}_{\gamma}^{\mathrm{SM}}$ on $\mathbf{A}_{33}^{\text {de }}$ and $\tan \beta$. For each example listed in Table 2 (and also for 17 other examples), we have plotted $\delta \mathcal{B}_{\gamma}$ keeping all the parameters but $\mathbf{A}_{33}^{d e}$ fixed. As one can see, SUSY contributions in our examples can enhance $\mathcal{B}_{\gamma}$ by up to $30 \%$ w.r.t. the $\mathrm{SM}$ prediction $\mathcal{B}_{\gamma}^{\mathrm{SM}}=$ $(3.15 \pm 0.23) \times 10^{-4}$ [30]. Moreover, up to $10 \%$ relative differences are observed between points with vanishing and maximal $\mathbf{A}_{33}^{d e}$. The current experimental world average yields $\mathcal{B}_{\gamma}^{\exp }=(3.43 \pm 0.22) \times 10^{-4}$ [31]. The observed significant variation of $\mathcal{B}_{\gamma}$ could lead to a possible verification of the model once the uncertainties get reduced.

Analogous plots for $\delta \overline{\mathcal{B}}_{s \mu} \equiv\left(\overline{\mathcal{B}}_{s \mu}^{\mathrm{MSSM}}-\overline{\mathcal{B}}_{s \mu}^{\mathrm{SM}}\right) / \overline{\mathcal{B}}_{s \mu}^{\mathrm{SM}}$ are shown in Fig. 9. All our sample results for $\overline{\mathcal{B}}_{s \mu}^{\mathrm{MSSM}}$ fall within the $1 \sigma$ band above the measurement $\overline{\mathcal{B}}_{s \mu}^{\exp }=$ $(2.9 \pm 0.7) \times 10^{-9}[32-34]$ and the branching ratio can be smaller by about $15 \%$ compared to the SM prediction $\overline{\mathcal{B}}_{s \mu}^{\mathrm{SM}}=(3.65 \pm 0.23) \times 10^{-9}[35]$. As far as $\overline{\mathcal{B}}_{d \mu}$ is concerned, it undergoes an almost identical alteration with respect to the SM. However, it remains in perfect agreement with the present experimental result $\overline{\mathcal{B}}_{d \mu}^{\exp }=\left(3.6_{-1.4}^{+1.6}\right) \times 10^{-10}[32-$ 34] within its large uncertainties. The experimental sensitivity would need to be improved by more than an order of magnitude to distinguish between the SM prediction $\overline{\mathcal{B}}_{d \mu}^{\mathrm{SM}}=(1.06 \pm 0.09) \times 10^{-10}[35]$ and the corresponding MSSM results for our sample points.

The three considered decays share the crucial property of being sensitive to supersymmetric contributions even if no sources of flavour violation beyond the CKM matrix are present. It follows from the fact that they are all chirally suppressed in the SM.

\section{Electroweak symmetry breaking}

The MSSM contains a large number of scalar fields. In a proper analysis of the electroweak symmetry breaking, one would need to prove that only the neutral Higgs fields acquire non-zero values in the global minimum of the MSSM scalar potential. However, it is well known that there exist large regions in the MSSM parameter space where other, deeper minima arise. At such minima, also sfermions develop nonvanishing vacuum expectation values.

In particular, along the direction in the MSSM scalar field space where

$\left|H_{1}\right|=\left|\tilde{s}_{L}\right|=\left|\tilde{s}_{R}\right|$,

a deeper, charge and colour breaking minimum arises when $A_{s}$ ( $\left.M_{\text {SUSY }}\right)$ is large. Actually, all our examples in Tables 2 and 3 strongly violate the stability condition [36]

$\frac{A_{i i}}{Y_{i i} \widetilde{m}_{i}}<O(1)$.

with $\tilde{m}_{i}$ defined by Eq. (11). Instead, we have

$\frac{A_{s}}{Y_{s} \tilde{m}_{2}}\left(M_{\mathrm{SUSY}}\right) \sim 10^{2}$.

However, the usual Higgs vacuum does not need to be absolutely stable. The standard viability condition is that the its lifetime must be longer than the age of the Universe. According to Ref. [37], such a condition is fulfilled when 

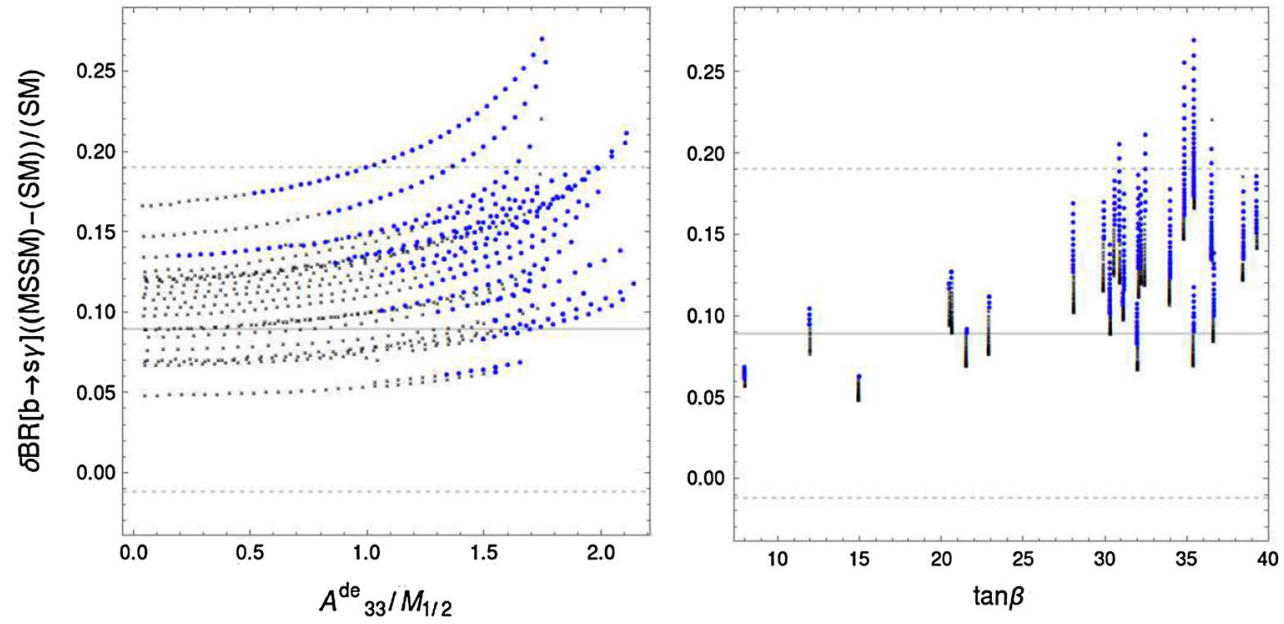

Fig. 8 Dependence of $\delta \mathcal{B}_{\gamma}$ on $\mathbf{A}_{33}^{d e}$ and $\tan \beta$. Points fulfilling $\mathbf{Y}^{d}\left(M_{\mathrm{GUT}}\right)=\mathbf{Y}^{e T}\left(M_{\mathrm{GUT}}\right)$ are marked in blue. The $1 \sigma$ experimental error band is represented by horizontal lines
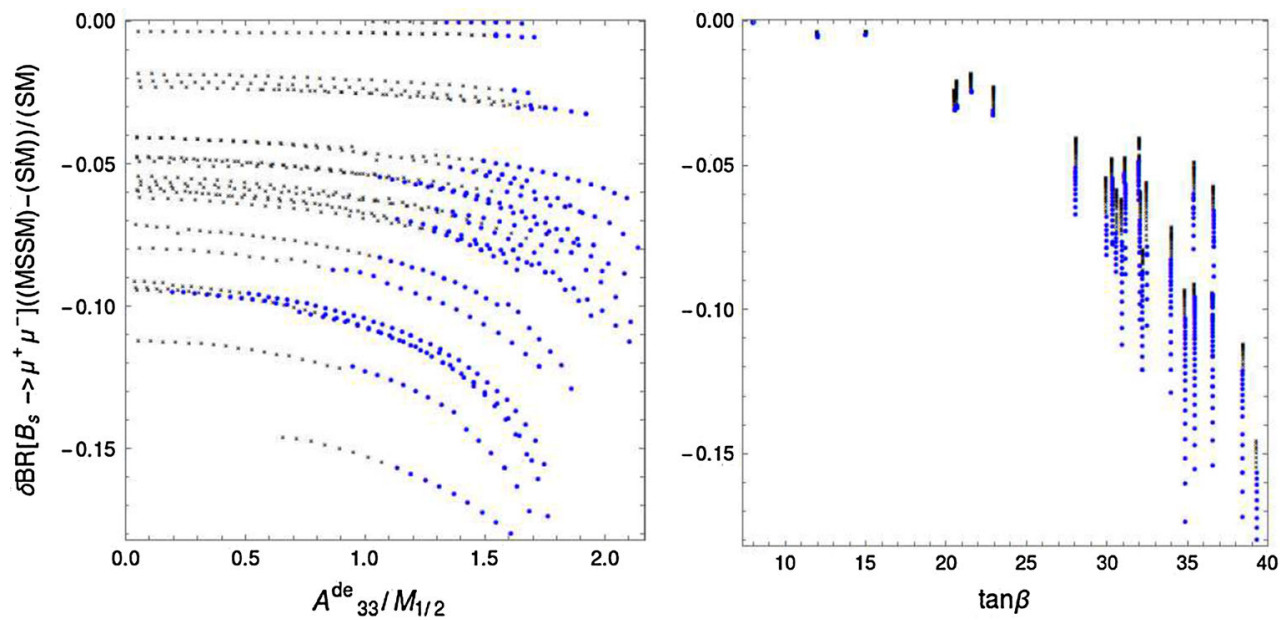

Fig. 9 Dependence of $\delta \overline{\mathcal{B}}_{s \mu}$ on $\mathbf{A}_{33}^{d e}$ and $\tan \beta$. For each point listed in Table 2, only $\mathbf{A}_{33}^{d e}$ has been varied. Points fulfilling $\mathbf{Y}^{d}\left(M_{\mathrm{GUT}}\right)=\mathbf{Y}^{e T}\left(M_{\mathrm{GUT}}\right)$ are marked in blue. The results for $\delta \overline{\mathcal{B}}_{d \mu}$ are practically identical

Table 6 Values of the diagonal entries of $A$ terms at scale $M_{S U S Y}$ of the points from Table 2

\begin{tabular}{llll}
\hline & $\frac{A_{d}}{\tilde{m_{1}}}$ & $\frac{A_{s}}{\tilde{m_{2}}}$ & $\frac{A_{b}}{\tilde{m_{3}}}$ \\
\hline 1 & -0.0064 & 0.49 & 1.26 \\
2 & -0.016 & 0.89 & 1.01 \\
3 & -0.041 & 1.56 & 0.38 \\
4 & -0.048 & 1.54 & 0.18 \\
\hline
\end{tabular}

$\frac{\mathbf{A}_{s}}{\widetilde{m}_{2}}<1.75$.

This requirement turns out to be satisfied in all our examples of Yukawa unification. One can verify this by inspecting Table 6 where the ratios $\frac{A}{\tilde{m}}$ have been presented for all the three generations.

\section{Conclusions}

Searches for supersymmetric particles during the first LHC phase have significantly constrained the MSSM parameter space. With heavier superpartners, the little hierarchy problem becomes more difficult, but the SUSY flavour problem is rendered less severe. Thus, if the MSSM is the proper lowenergy theory, one should consider its possibly non-trivial flavour structure. To check its consistency with grand unification, we need to understand all the factors involved in fulfilling the GUT boundary conditions for the Yukawa matrices. Given only few existing analyses of the MSSM threshold corrections' impact on the Yukawa matrix unification, we performed an update that takes the recent experimental data into account.

Our article provided examples of successful $S U(5)$ Yukawa unification that is consistent with the current exper- 
imental bounds in a scenario where all the soft terms are flavour-diagonal. It did come at a price. An adjustment of the down-quark Yukawa couplings governed by the $A$-terms of the sfermion mass size led to a conclusion that the usual Higgs vacuum becomes metastable. However, given its long enough lifetime, such a situation is still phenomenologically viable.

Given the viability of the $S U(5)$ boundary conditions on MSSM Yukawa couplings at $M_{\mathrm{GUT}}$, it might be interesting to investigate the case of $S O(10)$. Unfortunately, the tiny $\frac{m_{c}}{m_{t}}$ ratio at the low scale is difficult to obtain in the minimal $S O$ (10) GUT framework. Eventually, $S O$ (10)-type unification could be achieved by employing almost complete cancellations of threshold corrections to $Y_{c}$ against tree-level terms, i.e. it would come at a price of considerable fine-tuning.

Different ways of explaining the Yukawa matrix unification are complementary. In a general case, both the MSSM threshold corrections and the GUT-scale higher-dimensional operators can be present. If a complete quantitative study of a specific GUT model were to be performed, all such options would need to be simultaneously taken into account.

We satisfied the Yukawa matrix unification constraint in possibly the simplest manner, by adjusting just three parameters. The remaining freedom in the choice of other soft parameters in this scenario gives it the advantage of modularity. Such a freedom is likely to facilitate satisfying additional phenomenological constraints (like the observed dark matter relic density) or fitting new observables that might prove relevant for future studies.

Acknowledgments The author would like to thank Andreas Crivellin, Christophe Grojean, Gian Giudice, Kamila Kowalska, Mikołaj Misiak and Ulrich Nierste for valuable discussions and sharing ideas. The hospitality of CERN and the Karlsruhe Institute of Technology is gratefully acknowledged. This work was supported in part by the Foundation for Polish Science International PhD Projects Programme co-financed by the EU European Regional Development Fund, by the Karlsruhe Institute of Technology and by the National Science Centre (Poland) research project, decision DEC-2011/01/B/ST2/00438.

Open Access This article is distributed under the terms of the Creative Commons Attribution License which permits any use, distribution, and reproduction in any medium, provided the original author(s) and the source are credited.

Funded by $\mathrm{SCOAP}^{3}$ / License Version CC BY 4.0.

\section{References}

1. S. Dimopoulos, H. Georgi, Nucl. Phys. B 193, 150 (1981)

2. H. Georgi, C. Jarlskog, Phys. Lett. B 86, 297 (1979)

3. S. Antusch, M. Spinrath, Phys. Rev. D 79, 095004 (2009). arXiv:0902.4644 [hep-ph]

4. S. Antusch, S.F. King, M. Spinrath, Phys. Rev. D 89, 055027 (2014). arXiv:1311.0877 [hep-ph]

5. D. Emmanuel-Costa, S. Wiesenfeldt, Nucl. Phys. B 661, 62 (2003). hep-ph/0302272

6. W. Martens, L. Mihaila, J. Salomon, M. Steinhauser, Phys. Rev. D 82, 095013 (2010). arXiv:1008.3070 [hep-ph]

7. W. Buchmuller, D. Wyler, Phys. Lett. B 121, 321 (1983)
8. L.J. Hall, V.A. Kostelecky, S. Raby, Nucl. Phys. B 267, 415 (1986)

9. J.L. Diaz-Cruz, H. Murayama, A. Pierce, Phys. Rev. D 65, 075011 (2002). hep-ph/0012275

10. T. Enkhbat, arXiv:0909.5597 [hep-ph]

11. B. Bajc, S. Lavignac, T. Mede, AIP Conf. Proc. 1604, 297 (2014). arXiv: 1310.3093 [hep-ph]

12. C.S. Aulakh, S.K. Garg, Nucl. Phys. B 857, 101 (2012). arXiv:0807.0917 [hep-ph]

13. C.S. Aulakh, I. Garg, C.K. Khosa, Nucl. Phys. B 882, 397 (2014). arXiv:1311.6100 [hep-ph]

14. A. Crivellin, L. Hofer, J. Rosiek, JHEP 1107, 017 (2011). arXiv:1103.4272 [hep-ph]

15. A. Crivellin, U. Nierste, Phys. Rev. D 79, 035018 (2009). arXiv:0810.1613 [hep-ph]

16. A. Crivellin, Phys. Rev. D 83, 056001 (2011). arXiv:1012.4840 [hep-ph]

17. A. Crivellin, J. Girrbach, Phys. Rev. D 81, 076001 (2010). arXiv:1002.0227 [hep-ph]

18. A. Crivellin, C. Greub, Phys. Rev. D 87, 015013 (2013). arXiv: 1210.7453 [hep-ph]

19. F. Brummer, S. Kraml, S. Kulkarni, JHEP 1208, 089 (2012). arXiv:1204.5977 [hep-ph]

20. B.C. Allanach, D.P. George, B. Gripaios, JHEP 1307, 098 (2013). arXiv:1304.5462 [hep-ph]

21. B.C. Allanach, D.P. George, B. Nachman, JHEP 1402, 031 (2014). arXiv:1311.3960 [hep-ph]

22. B.C. Allanach, Comput. Phys. Commun. 143, 305 (2002). arXiv:hep-ph/0104145

23. A. Crivellin, J. Rosiek, P.H. Chankowski, A. Dedes, S. Jaeger, P. Tanedo, Comput. Phys. Commun. 184, 1004 (2013). arXiv:1203.5023 [hep-ph]

24. A. Fowlie, M. Kazana, K. Kowalska, S. Munir, L. Roszkowski, E.M. Sessolo, S. Trojanowski, Y.L.S. Tsai, Phys. Rev. D 86, 075010 (2012). arXiv:1206.0264 [hep-ph]

25. K. Kowalska, JHEP 1409, 139 (2014). arXiv:1406.0710 [hep-ph]

26. F. Feroz, M.P. Hobson, M. Bridges, Mon. Not. R. Astron. Soc. 398, 1601 (2009). arXiv:0809.3437 [astro-ph]

27. J. Beringer et al., Particle Data Group, Phys. Rev. D 86, 010001 (2012)

28. W. Porod, Comput. Phys. Commun. 153, 275 (2003). hep-ph/0301101

29. W. Porod, F. Staub, Comput. Phys. Commun. 183, 2458 (2012). arXiv:1104.1573 [hep-ph]

30. M. Misiak, H.M. Asatrian, K. Bieri, M. Czakon, A. Czarnecki, T. Ewerth, A. Ferroglia, P. Gambino et al., Phys. Rev. Lett. 98, 022002 (2007). arXiv:hep-ph/0609232

31. Y. Amhis et al., [Heavy Flavor Averaging Group Collaboration], arXiv:1207.1158 [hep-ex], and online update at http://www.slac. stanford.edu/xorg/hfag

32. CMS and LHCb Collaborations, EPS-HEP 2013 European Physical Society Conference on High Energy Physics, Stockholm, Sweden, 2013, conference report no. CMS-PAS-BPH-13-007, LHCbCONF-2013- 012. http://cds.cern.ch/record/1564324

33. S. Chatrchyan et al., CMS Collaboration, Phys. Rev. Lett. 111, 101804 (2013). arXiv:1307.5025 [hep-ex]

34. R. Aaij et al., LHCb Collaboration, Phys. Rev. Lett. 111, 101805 (2013). arXiv:1307.5024 [hep-ex]

35. C. Bobeth, M. Gorbahn, T. Hermann, M. Misiak, E. Stamou, M. Steinhauser, Phys. Rev. Lett. 112, 101801 (2014). arXiv:1311.0903 [hep-ph]

36. J.A. Casas, A. Lleyda, C. Muñoz, Nucl. Phys. B 471, 3 (1996). arXiv:hep-ph/9507294

37. F. Borzumati, G.R. Farrar, N. Polonsky, S.D. Thomas, Nucl. Phys. B 555, 53 (1999). hep-ph/9902443 